\title{
Speculative Practices: Utilizing InfoVis to Explore Untapped Literary Collections
}

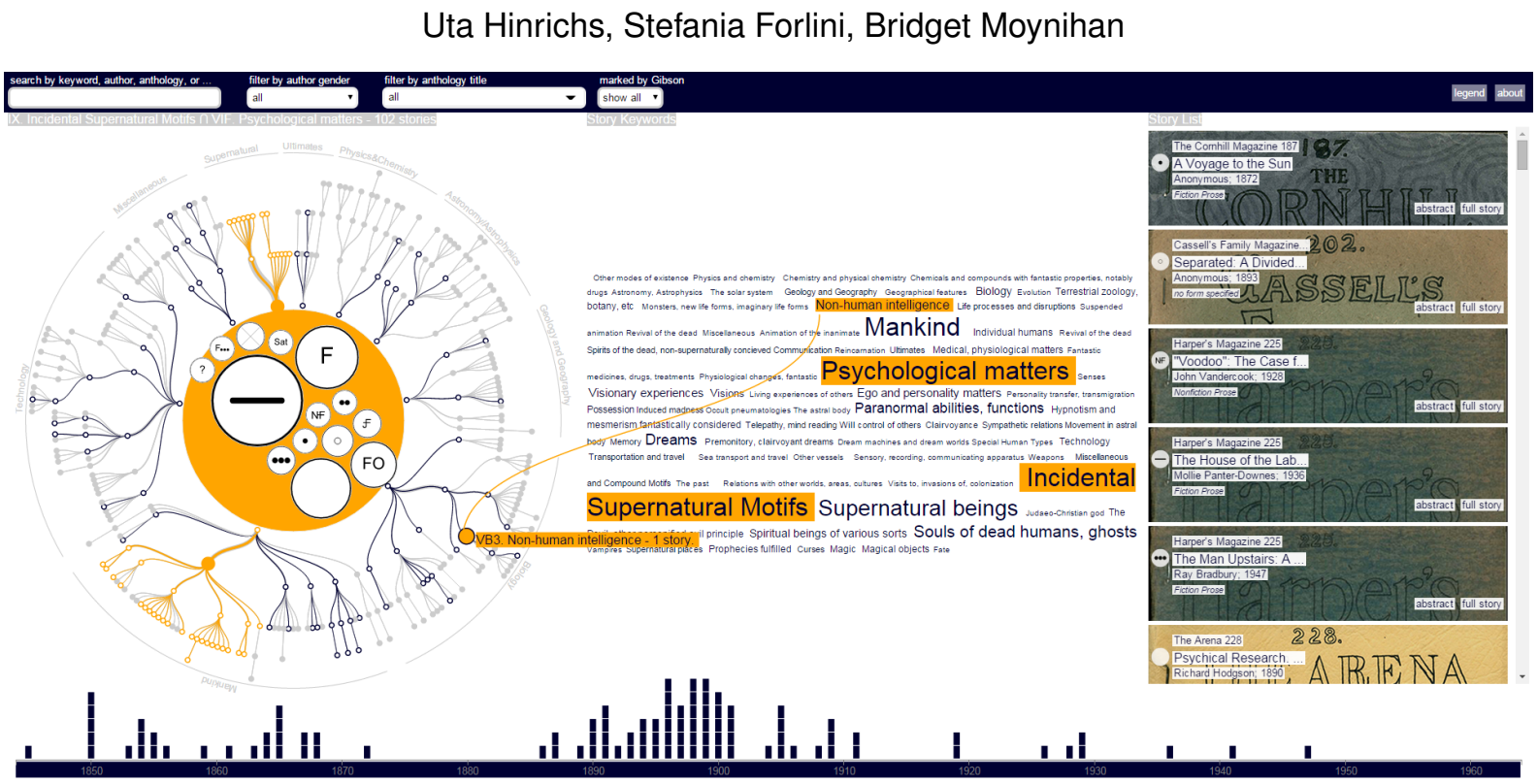

Fig. 1: The Speculative W@ nderverse of the B. Gibson Anthologies.

\begin{abstract}
In this paper we exemplify how information visualization supports speculative thinking, hypotheses testing, and preliminary interpretation processes as part of literary research. While InfoVis has become a buzz topic in the digital humanities, skepticism remains about how effectively it integrates into and expands on traditional humanities research approaches. From an InfoVis perspective, we lack case studies that show the specific design challenges that make literary studies and humanities research at large a unique application area for information visualization. We examine these questions through our case study of the Speculative W@nderverse, a visualization tool that was designed to enable the analysis and exploration of an untapped literary collection consisting of thousands of science fiction short stories. We present the results of two empirical studies that involved general-interest readers and literary scholars who used the evolving visualization prototype as part of their research for over a year. Our findings suggest a design space for visualizing literary collections that is defined by (1) their academic and public relevance, (2) the tension between qualitative vs. quantitative methods of interpretation, (3) result- vs. process-driven approaches to InfoVis, and (4) the unique material and visual qualities of cultural collections. Through the Speculative W@nderverse we demonstrate how visualization can bridge these sometimes contradictory perspectives by cultivating curiosity and providing entry points into literary collections while, at the same time, supporting multiple aspects of humanities research processes.
\end{abstract}

Index Terms_-Digital Humanities, Interlinked Visualization, Literary Studies, Cultural Collections, Science Fiction.

\section{INTRODUCTION}

Information visualization has started to play a role in humanities research. This is visible in the increasing number of case studies [1, 20, 38], tools [37, 43, 44, 50, 51] and publications that discuss InfoVis as a new research method in the humanities [13, 23, 25]. This vivid discussion, which is interwoven with both excitement and skepticism $[13,19,25,26,39]$, raises several questions relevant to humanities as well as InfoVis research. How can InfoVis facilitate research

- Uta Hinrichs is with the SACHI Group, University of St Andrews, UK E-mail:uh3@st-andrews.ac.uk.

- Stefania Forlini is with the Department of English, University of Calgary. E-mail: sforlini@ucalgary.ca.

- Bridget Moynihan is with the Department of English, University of Calgary.Email: bcmoynih@ucalgary.ca.

Manuscript received 31 Mar. 2015; accepted 1 Aug. 2015; date of publication xx Aug. 2015; date of current version 25 Oct. 2015. For information on obtaining reprints of this article, please send e-mailto: tvcg@computer.org. approaches and processes in the humanities? What types of insights can be supported? How can visualization tools integrate into existing research approaches in the humanities such as archival work and close reading? And finally: What makes the humanities a unique research context for information visualization, and what are the challenges of designing InfoVis tools to facilitate humanities research? We begin to address these questions in this paper, based on the case study of the Speculative W@nderverse-a visualization that we designed to facilitate the literary analysis and exploration of a vast literary collection of early science fiction.

The Speculative W@nderverse is the result of an interdisciplinary design process that involved literary scholars and information visualization experts. This process, as well as our findings from two empirical studies that investigated how general-interest readers and literary scholars explored the visualization, suggest a design space for information visualization within a literary studies context that is defined by: (1) an audience that ranges from general-interest readers and fans as amateur experts to academics interested in in-depth literary analysis and interpretation, (2) an approach that reflects a tension between 

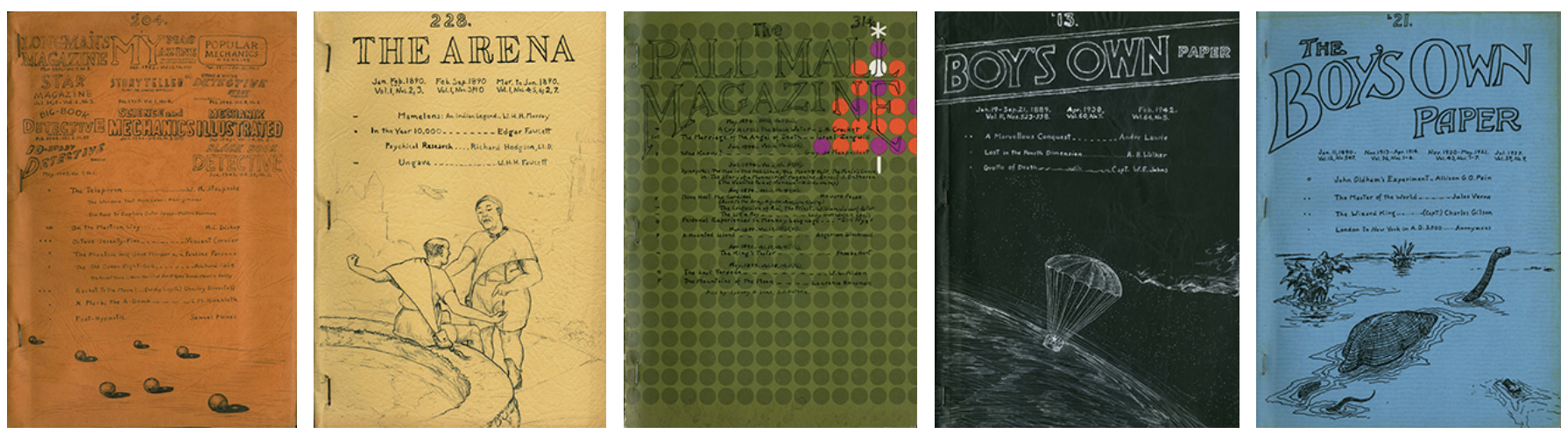

Fig. 2: Selection of five of Gibson's Anthologies, showing their unique handcrafted covers.

quantitative analysis and qualitative interpretation of literary collections, (3) the role of visualization as a process rather than a means to an end (result vs. process), and (4) the material and visual qualities of a literary collection that influence interpretation approaches.

Overall, these dimensions can be interpreted as high-level considerations to facilitate the design of visualizations in the context of literary research while promoting productive synergies between literary scholars and related disciplines, amateur experts, and general-interest readers. They can also be applied to critique and evaluate existing visualizations in the humanities context and may facilitate a dialogue between visualization experts and humanities scholars.

Our paper is written predominantly for an InfoVis audience to shed light on the research processes in literary studies and the humanities at large, and to inform the design and future research around information visualization in this context. It will be also useful for researchers in the (digital) humanities interested in integrating visualization into their research processes. Furthermore, it presents one example of how to conduct interdisciplinary collaborations in the literary context. To summarize, this paper makes the following contributions:

- The definition and discussion of a design space which outlines four productive challenges of facilitating literary research through information visualization, including considerations of the audience, humanities' research approaches, the role of visualization, and material and visual qualities of literary collections.

- The Speculative W@nderverse as a visualization case study that exemplifies one interpretation of this design space and that illustrates how some of its seemingly opposite design tensions can be bridged.

- An overview of the types of insights that visualizations such as the W@nderverse can promote among general-interest readers and academics, e.g., providing entry points to complex and untapped literary collections, confirming hypotheses, refining research questions, and negotiating quantitative and qualitative interpretation.

- A critical discussion of our approach, practical considerations, and future research questions regarding visualizing literary collections for scholarly and general-interest exploration that we identified through our case study.

We start with an overview of the Gibson Anthologies, the literary collection which forms the basis for our work. We then provide a summary of related research and introduce the dimensions, which compose a design space for visualization in a literary studies context. This is followed by a description of the Speculative W@nderverse as one interpretation of this space and our findings from two empirical studies. We end with a critical discussion of our approach, its practical implications and the future research questions it raises.

\section{Research Background: The Gibson Anthologies}

Our interdisciplinary research is grounded in a wide, and largely untapped, literary collection, The Bob Gibson Collection of Speculative Fiction, compiled by the Canadian collector and avid science fiction fan Bob Gibson (1908-2001). Gibson's collection, which is certified as Canadian Cultural property [4], contains approximately 40,000 science fiction books, magazines, and periodicals. We focus on a particularly unique part of this magnificent collection: the more than 890 speculative fiction anthologies that were handcrafted by Gibson.

These anthologies are the result of Gibson's painstaking collection and curation of a wide range of science-fictional materials from magazines published between the 1840s and 1990s, including prose and verse, fiction and nonfiction, short stories, comics, serialized novels, and many illustrations. The anthologies can be read as an expansive attempt to archive a cultural imaginary of science fiction and speculation in many aesthetic forms as these genres developed over time. Moreover, Gibson hand-illustrated many of his covers with original drawings (see Fig. 2), and provided a hand-written table of contents for each anthology, which includes symbols through which he rated the "science fiction content" of his collected items (see Fig. 3).

The breadth of Gibson's anthologies, together with his unique classification system, offer an opportunity to challenge and reinvigorate existing categorizations of science fiction that typically neglect the experimental, periodical-based origins of the genre. For example, the range of authors that Gibson compiled in his anthologies includes many previously unknown women writers and so raises questions about early science fiction history, which is typically defined as evolving through the work of men. Furthermore, deciphering Gibson's symbols (see Fig. 3), to which he has left no key, may challenge and revise current systems of generic classification. Although our deciphering is still in progress, we describe how information visualizations have facilitated our analysis process and led to some initial findings.

It is not only their range and content that makes the Gibson Anthologies so complex, but also their visual and material qualities. Gibson cut stories and other content he deemed relevant from magazines and assembled these using glue and scrap paper. Every anthology is physically and visually unique in its cover page illustrations, lettering, material, size, and binding. The anthologies will be fully digitized eventually, but their material qualities complicate computational processing. While we considered text mining to extract metadata automatically from each anthology item, we decided against this approach for our initial case study. Text mining is powerful but, even on more standardized text documents, can lead to considerable noise in the extracted data, often due to OCR errors. We therefore decided to follow a common approach in digital humanities projects, namely, to manually extract metadata from a subset of 72 anthologies that contain the earliest items (1513 items in total). This enabled us to conduct an initial exploration of the Gibson Anthologies and their general potential to

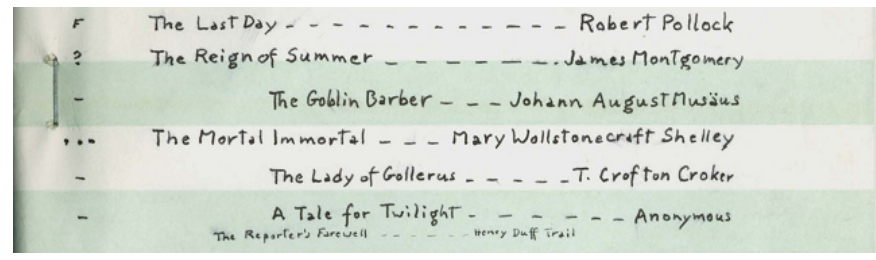

Fig. 3: Anthology cover: table of contents and symbols for each item. 
further science fiction research. From this subset, 52 anthologies have been digitized and are available through a university library website ${ }^{1}$.

The collection of metadata was conducted by a team of English literature scholars and students who read every item in our anthology subset. For each anthology item, we record title, author name, author gender (if known), publication or reprint year, source magazine, Gibson's symbol, corresponding anthology, literary form, abstract (written by our researchers), and a list of keywords describing the item's content. We work with an existing classification system introduced by Bleiler [2], a widely-recognized authority in science fiction criticism who developed a keyword hierarchy based on nine major motifs (e.g., astronomy/astrophysics, mankind, and technology) to describe content in early science fiction stories. This system enables us to explore the themes within our anthologies, and to compare Gibson's classifications with those of Bleiler. Our metadata is stored in a relational database that forms the basis for the Speculative W@ nderverse.

We started our exploration of the Gibson Anthologies with two questions: What can this collection reveal about the historical evolution of science fiction? And: How can Gibson's editorial choices and approach both to collecting and to science fiction be characterized? Before we describe the W@nderverse visualization, we outline related work that inspired our process, and discuss the productive tensions unique to designing visualizations in the humanities context.

\section{Related Work}

Visualizing cultural and document collections for pleasurable exploration and analysis are active topics in information visualization. Many sophisticated techniques and systems have been introduced in this area, such as word clouds [50, 51], parallel tag clouds [9], word trees [52], and phrase nets [48], to name a few. Some of them are being applied by humanities scholars. Moretti, for example, makes extensive use of static graphs and network visualizations [27], Plaisant et al. use an interactive visualization, which features scatterplots to analyze Emily Dickinson [31], and word clouds are frequently used to investigate emergent themes in large and small-scale document corpora (see Jockers [25, chpt. 8]). Parallel tag clouds [9] have been used in the context of environmental history to make geographic trends in commodity trading interactively explorable $[20,14]$.

Powerful visualization systems conceived by the visualization and digital humanities communities support the exploration and analysis of text-based collections. JigSaw, for example, is a visual analytics system to support sense-making across large document collections [45]. FeatureLens enables the exploration of patterns within text-based collections at different levels of granularity [10,8]. ManyEyes supports rapid data exploration and discussion through customizable web-based visualizations [49]. The visualization platform Gephi enables the representation of networks and hierarchical data ${ }^{2}$. Sinclair and Ruecker's web-based Voyant Tools feature several visualization components to analyze and explore textual collections [43]. $\mathrm{TAPoR}^{3}$, a meta-tool that provides an overview of existing tools for textual analysis and interpretation, shows the rapidly increasing number of tools in this area.

While a comprehensive discussion of visualization tools and techniques used with cultural and document collections in a humanities context is beyond the scope of this paper (see Jänicke for an overview [23]), there are certain trends that stand out. First, many tools feature coordinated views, wherein multiple visualizations show a range of metadata and relations within a collection. This strategy has been extensively discussed in the visualization community to facilitate investigative analysis [45, 53], the exploration of news articles [11], book collections [46], and artworks [21]. Studies have shown that the strategy of coordinated views is useful for the analysis of large and complex document collections, both in professional $[5,18]$ and casual contexts [11, 21]. Thudt et al. highlight this strategy as particularly effective for supporting open-ended exploration and serendipitous discoveries [46]. There is also a trend toward visualization systems that support exploratory approaches to investigating data collec-

\footnotetext{
${ }^{1}$ http://contentdm.ucalgary.ca/cdm4/browse.php?CISOROOT=/gcsf

${ }^{2}$ http://gephi.github.io/

${ }^{3}$ http://www.tapor.ca/docs? name=tour
}

tions. Through the notion of the "Flaneur", Dörk et al. propose a more experimental and playful approach to information exploration. Whitelaw highlights the importance of "generous interfaces" to promote and facilitate the exploration of cultural collections [54].

Typically, case studies discussed in the context of digital humanities focus on the novel design and functionality of visual tools or the results provided by these. Meanwhile in the visualization community long-term research processes and outcomes supported by visualization have been discussed in the area of journalism [5] and in casual scenarios [21]. What we lack are in-depth and long-term studies that show how information visualization can facilitate humanities research processes and how such visualizations integrate into traditional approaches such as archival work and close reading. Adapting wellestablished existing visualization techniques to the needs of a specific collection, we present the W@nderverse as part of a case study to illustrate in detail how a visualization prototype can be integrated into literary research processes. In addition, we start to map out the design space and challenges that emerge when designing visualizations in the context of cultural and/or literary collections.

\section{Visualization in the Humanities: The Design Space}

Through our interdisciplinary process of designing a visualization that would adequately represent the Gibson Anthologies for literary analysis and public exploration, we discovered unique constraints and productive tensions in this context that InfoVis has to address. We introduce these as characteristics of a design space that applies to research on literary collections specifically, but also to humanities research at large. This space differs from related application contexts that have previously been discussed in the visualization research community, such as casual or artistic scenarios [33], libraries [46], museums [3, 21], or journalism [5] by drawing from and expanding recent digital humanities discussions that seek to address critically the potential of information visualization in their research [13, 16, 17, 25, 27]. We posit four dimensions that characterize this space-the audience, research approaches within the humanities (quantitative analysis vs. interpretation), the role of visualization (result vs. process), and considerations on material and visual aspects of the source material. We show how each dimension spans a continuum between different, seemingly opposite, concepts. Our design dimensions can be understood as high-level considerations for designing and evaluating visualizations in the context of literary studies and humanities research at large. Last but not least, the dimensions raise questions for future research.

\subsection{Audience: Scholars, Fans \& General-Interest Readers}

Literary collections are of interest to both academic and non-academic audiences. The same work of literature can be approached for pleasure by general-interest readers, fans, and other non-academic experts, as well as by academic scholars who examine the ways in which literature reflects on and actively structures human history and society. With the digitization of literary collections, digital humanities researchers increasingly recognize an opportunity and a responsibility to make cultural collections and their related research available for both fellow scholars and a public audience (see Hayles for examples [19, chpt.2]), while collaborations between scholars and non-academic readers are also being initiated, for example, through crowdsourcing [15] or participatory approaches (e.g, [34]). Although digital humanities projects typically welcome public outreach, we know of no case study in the context of literary research where both public and academic audiences have been explicitly considered. Similarly, while case studies in information visualization have shown their suitability for casual, public audiences (e.g., [3, 21, 46]) on the one hand and for domain experts on the other (e.g., [5, 18], to name a few), few address simultaneously a range of expert and casual audiences (see $[18,29]$ for rare examples).

The challenge that our project undertakes is to design visualizations that can support the analysis and interpretation of literary collections, while potentially still providing a point of entry to the general public and fans, who may be interested in, for example, learning about the character of the collection in order to discover unknown and/or unusual readings and visual materials. Of course, it is possible to design separate tools that satisfy the unique characteristics of different 
audiences; however, through studying the Speculative W@nderverse in both public and academic settings, we found similarities across our target groups in their initial approaches to exploring a complex and largely unknown literary collection such as the Gibson Anthologies. Moreover, even our work on these anthologies can be considered an asynchronous collaboration with Gibson as a science fiction fan and collector. To facilitate opportunities for collaborations and synergies beyond academic boundaries, we argue for the design of hybrid visualizations that can address multiple audiences and therefore promote a participatory culture [24] that bridges communities of interested readers, fans, and academics. This approach is particularly fruitful for untapped literary collections where a broad exploration from multiple (e.g., amateur expert and academic) perspectives can further initial research questions and give the collection a new public presence.

\subsection{Approach: Quantitative Analysis vs. Interpretation}

Computational methods, including information visualization, have the potential to facilitate the study of larger document collections, literature, photographs, or other cultural artefacts from a humanities perspective $[25,27,42]$. The humanities repertoire of traditional research methods, many of which depend on close reading or the careful study, analysis, and interpretation of a small number of selected individual texts [25], is expanding to include what Moretti has named distant reading $[27,28]$ - macro-level approaches to large-scale literary collections, using quantitatively-based methods of analysis. Quantitative approaches to literature have not remained without critique, as they seem to suggest a fundamental shift from traditional constructivist practices that are based on fine-grained, qualitative observation and critical interpretation $[13,25]$, toward positivist approaches where the complexity and ambiguity of literary forms is reduced, even impoverished, in favour of abstraction and quantification [13, 26].

However, the interest in quantitative analyses of literary texts (seen by some as typical of the first wave of digital humanities), continues to be relevant even in the so-called second wave, which is described as "qualitative, interpretive, experiential, emotive, generative in character [boldface in original]" [39]. In fact, digital humanities scholars increasingly call for an integrated use of both quantitative and qualitative approaches, which holds the synergistic potential of combining these vastly different approaches to humanistic study without losing sight of "the Humanities' core methodological strengths: attention to complexity, medium specificity, historical context, analytical depth, critique and interpretation" [39]. For example, Kirshenbaum speaks of "rapid shuttling" between quantitative information and close reading [19]. Ramsay proposes "algorithmic criticism," which makes use of computational quantitative analyses without privileging their objectivity, and instead insisting on the meaning-making/interpretive practices of more traditional humanistic study [35]. Similarly, Jockers proposes a hybrid approach, which he calls macroanalysis [25]. Finally, even Moretti, well-known for courting controversy when he called on literary scholars to stop reading [27], bases his arguments on quantitative analyses, while remaining guided by a formalist approach to literary study and the expansive review of existing literary criticism (that employs close reading). In other words, it is important to notebut not to overstate - the tension between quantitative and qualitative approaches; the possibilities for synergy are actively being explored.

We propose that information visualization is uniquely suited to foster the kind of synergy sought by many digital humanities scholars, even if its potential in humanities research has yet to be systematically studied. Through interactivity and visual representations, visualization can enable multiple perspectives on literary collections, promoting a variety of possible interpretations, raising questions, and inspiring creative thinking. As we illustrate through the Speculative W @ nderverse, we see the potential of information visualization in the literary context as fostering interactive experiments that are not a means to an end but a starting point to explore, interpret, and discuss literary collections.

\subsection{The Role of Visualization: Result vs. Process}

Some humanities scholars use visualization techniques primarily at the end of the research process to communicate patterns and trends that support an argument. Moretti's use of standard static visualizations to formulate and demonstrate his argument on the evolution of literary history is a good example of such visualization use [27, 28]. While communication is obviously an important aspect of information visualization, we argue for its potential to become an active and dynamic part of humanities research processes.

The increasing number and types of interactive visualization tools that are being developed within the digital humanities community (see [23] for an overview) indicate that at least some researchers recognize the value of integrating visualization into humanities research processes. Some recent studies even focus on experimental interactions with visualization tools (see, for example, [6]), which are recognized as inviting researchers to play with data and possible interpretations [7]. Unfortunately, as a recent study shows, such tools are not widely used [17]. This low adoption of tools into the research process itself is likely due in part to a combination of the previously mentioned tendency to use tools for result communication and insufficient consideration of usability and usage scenarios [40]. This highlights the importance of designing visualizations that invite exploration and that are driven by the needs and expectations of a broad range of prospective users (not all of whom will be technically savvy or inclined toward learning complex digital tools [17]). Collaborations between visualization experts and humanities scholars are essential to design such visualizations that integrate into and enrich traditional humanities research processes beyond the quantitative communication of results.

\subsection{Material and Visual Qualities: Identity vs. Utility}

Visualizing literary collections based on metadata as abstractions and reductions from the actual literary sources runs the risk of obscuring their unique visual and material qualities. Previous work in InfoVis and HCI has highlighted the importance of visual aesthetics in particular to promote curiosity, willingness to explore information spaces, and inspirational experiences that lead to meaningful and serendipitous discoveries (e.g, $[12,21,46,47])$. When digitizing any collection, but especially cultural collections, particular attention needs to be paid to aesthetic properties that extend beyond the visual. The aesthetics of a piece of literature, for example, may be expressed through the text itself, as well as through its typography, page layout, illustrations that go with the writing, the paper on which the work is printed or written, and the cover and binding of the book in which it is included. In humanities' research, it is not only the content which is analyzed and interpreted but also the artifactual form in which it is presented. Obscuring these forms in the visualization potentially impoverishes interpretation. Consequently, researchers in the digital humanities have started to discuss how visualizations function as "digital artifacts", reflecting their physical counterparts in meaningful ways and actively promoting interpretation [16]. Some visualization case studies can be regarded as experiments in developing such digital artifacts [21, 32].

Such approaches, however, seem to stand in contrast to the idea of developing visualization tools that have a broad utility in the humanities - such tools necessarily offer standard visual representations leaving less room to accommodate unique, visual and material characteristics of an individual collection. This is where we see space for future research. How can unique visual and material qualities of cultural collections be incorporated into visual representation techniques? Rather than providing answers to this question, the Speculative W@nderverse in its current stage and its unique underlying collection highlight the need for further research in this area.

\section{The Speculative W@Nderverse}

The Speculative W@anderverse is an interlinked visualization that consists of four main views: the Item View, the Keyword Cloud, the Keyword Hierarchy, and the Timeline. In addition, we provide a filter panel for refining these views based on particular parameters.

\subsection{Item View}

The Item View shows a list of science-fictional items, including their title, author, publication or reprint year, literary form, and a stylized version of the symbol that Gibson assigned to the item (see Fig.1, 5, and 6, right). A direct link leads to the item's abstract, which opens directly within the Item View (see Fig. 6). Another link provides direct access to the digitized version of the item-an important feature 


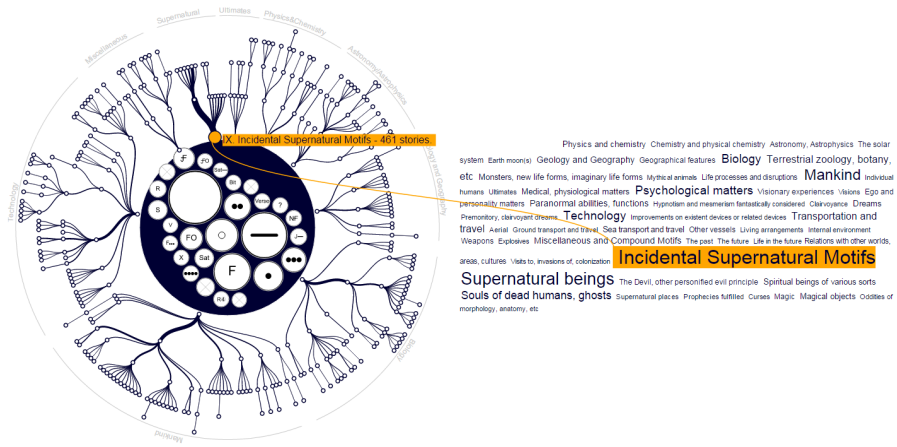

Fig. 4: Keyword Cloud \& Hierarchy with mouse-hover over a motif.

for scholars, fans, and a general-interest audience. The background of each list item shows the cover of its corresponding anthology. This integrates some of the unique anthologies' material and visual qualities into the visualization and, at the same time, makes it easy to identify which items belong to the same anthology. By default, items are grouped by anthology. Clicking the anthology cover provides a detailed full-screen view of the cover itself. Clicking the cover again closes this view and leads back to the visualization.

\subsection{Keyword Cloud \& Hierarchy View}

The Keyword Cloud and Hierarchy View reflect on the anthologies' content in relation to established motifs in early science fiction [2]. The Keyword Cloud provides an overview of the Bleiler keywords that correspond to each Gibson item (see Fig. 4, right). In this representation, the keywords' hierarchical structure is neglected in favour of a familiar overview of the themes present in the anthologies [50].

Complementing the Keyword Cloud, the Hierarchy View is based on a radial tree layout ${ }^{4}[36]$ that illustrates the keywords' hierarchical character (see Fig. 4, left). Each node represents a Bleiler keyword: the higher up (i.e., the more general) the keyword in the hierarchy, the smaller the distance of the corresponding node to the center of the tree. Edges between nodes represent their connection in the hierarchy; the weight of each edge represents the number of items corresponding to its target node. The radial tree layout was chosen consciously to ensure that no motif is presented as hierarchically and structurally superior to any other. Where Bleiler introduced a preemptive ordering by numbering the motifs, we instead allow the content of the Gibson anthologies to emphasize particular motifs. In this way, topical trends within the anthologies become visible. For example, the "Supernatural" branch has a strong representation amongst the Gibson items, with most items corresponding to "Supernatural Beings" (see Fig. 4, left). These visual choices exemplify how quantitative analysis and qualitative interpretation are combined in the W@ nderverse visualization.

While the familiar style of the Keyword Cloud caters to a casual audience or scholars with a more general interest in the Gibson anthologies, the Hierarchical View is useful for scholars that are familiar with the categorization and structure of early science fiction motifs (audience). We highlight the link between both views through a line that connects keywords in the Keyword Cloud and Hierarchy View upon hover. This also eliminates the problem of permanently labeling tree nodes in the Hierarchical View, which caused visual clutter.

Selecting a keyword/node in either the Keyword Cloud or Hierarchy View filters the Gibson items down to those corresponding to the active keyword: all views are updated in the Keyword Cloud and Hierarchy View with the Keyword Cloud only showing keywords that share at least one anthology item with the currently selected keyword. In the Hierarchy View, a selected keyword node is enlarged and its branch is highlighted in orange. Keyword nodes that intersect with the active keyword through Gibson items are shown in blue. Keywords that do not have any intersection with the active keywords are grayed out and become unselectable. For example, the selection of the keyword "Terrestrial zoology, botany, etc" highlights the corresponding branch in orange (see Fig. 5). This theme intersects with all other main themes in the Bleiler hierarchy except for "Ultimates", but many of the

${ }^{4}$ http://bl.ocks.org/mbostock/4063550 lower-level keywords and their corresponding branches are grayed out, which means there is no intersection between these themes and the selected keyword. In this way, the context of the hierarchy is preserved while preventing selections that produce empty results. It is possible to select multiple keywords to explore their intersections (see Fig. 1). Re-selecting an already active keyword removes it as a filter. Again, as with any selection, the other views are updated immediately.

As we will discuss later, these lightweight interactions enable a fluid interrogation of the collection's content and provide entry points for analysis and interpretation. The visual features suggest quantitative patterns; the text elements and chosen layout invite qualitative interpretation and interrogation (quantitative analysis vs interpretation; visualization as process vs. result).

\subsection{Symbol Cluster}

As stated earlier, Gibson did not leave a key for the symbols he assigned to most of his collected science-fictional items. Gibson's own marginalia, scattered throughout the anthologies, support the hypothesis that these symbols correspond to themes, genres and/or a ranking system. To explore this further, we integrated an overview of the symbols into the Hierarchy View (see Fig. 5). The Symbol Cluster in the center of the radial tree shows the number of symbols corresponding to the currently set filters (e.g., Bleiler keywords). With no filters set, an overview of all symbols is shown (see Fig. 4). Many items in our subset were not assigned any symbol by Gibson, noted in the visualization by a blank circle. In some cases, this is because Gibson chose not to assign a symbol, and in others because the item is an accidental inclusion in the anthology, e.g., an advertisement that appears on the back page of an item selected for the anthology. Since even such accidental inclusions speak to the character of the anthology, we decided to include them into our dataset, although they can be filtered out (quantitative analysis vs. interpretation).

Symbols are updated according to the selected filters to enable explorations of connections between particular Bleiler keywords and their corresponding symbols. For example, Figure 5 shows all symbols connected to the keyword branch "Incidental Supernatural Motifs". In addition, individual symbols can be selected, which updates all other visualizations to include only those items that correspond to the selected symbol (visualization as process vs. result; see Fig. 6).

\subsection{Timeline View}

The Timeline View shows the temporal distribution of Gibson items, based on their publication or reprint year. Each rectangle in the timeline represents an individual item (see Fig. 1). Linked to the other visual views the timeline always reflects on the currently active filters. Acting itself as a temporal filter, it can be adjusted like a slider to select a particular time period. This updates the other views accordingly (see Fig. 5). For general-interest audiences, this view provides a temporal context of the collection. For scholars, it enables focusing on collection items of particular time periods of interest (audience).

\subsection{Other Filtering Mechanisms}

The Speculative W@nderverse also features more traditional search and filter mechanisms. People can perform a free-form search that enables searching by keywords (beyond the Bleiler themes), authors, anthologies, and source magazines. An auto-complete function suggests possible options as people type in search terms. Furthermore,

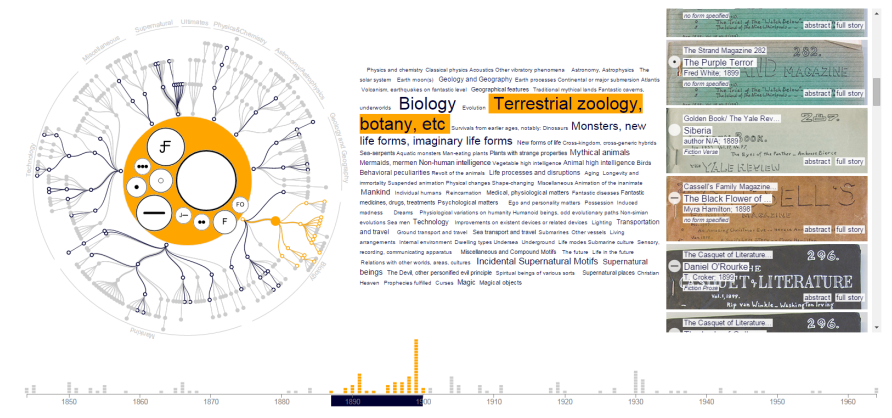

Fig. 5: Intersecting keyword branches (blue) in the Hierarchy View. Here, the Timeline View acts as an additional temporal filter. 


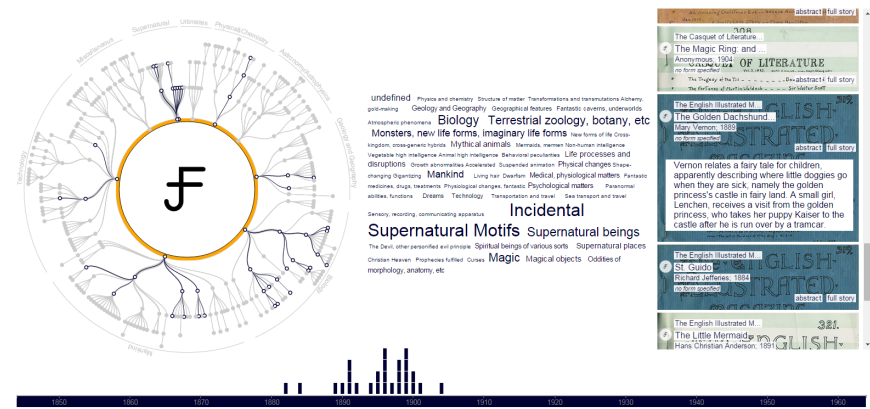

Fig. 6: Filtering keywords and items based on individual symbols.

three drop-down menus allow a filtering by author gender, anthology title, and items explicitly marked for inclusion in the anthologies by Gibson (thus enabling filtering of the previously mentioned accidental inclusions). All filters are integrated into a filter bar above the main visualizations (see Fig. 1). If a filter is active, it is highlighted in orange. The choice and design of filters was driven by the interests of our scholars without trying to interfere with the needs of a general-interest audience that may be opposed to an interface that seems visually complex and difficult to grasp (audience). Furthermore, we found that the coordinated views that act as visual filters, in combination with the search and filter bar, became crucial elements that supported both qualitative analysis and interpretation, as well as dynamic interaction with the visualization that easily integrated into our scholars' research processes (visualization as process vs. result).

We conducted two empirical studies to investigate how academic and general-interest audiences experience our visualization-based approach to the Gibson Anthologies, and how this reflects on the design space for information visualization in a humanities context.

\section{Evaluating the Speculative W@NDERVERse}

We studied the Speculative W@nderverse in two different contexts. First, we conducted two open-house events where we exhibited an early version of the visualization and gathered feedback from generalinterest readers and scholars via questionnaires. Second, the literary scholars of our team took detailed notes of their explorations of the anthologies, both in the archives and through their use of the W@nderverse, keeping track of their hypotheses, evolving research questions, and general thoughts as the visualization evolved.

\subsection{Open House Events}

Our two open house events took place at a university library (two days) and public library (one day). At the university library, we exhibited an early version of the W@nderverse on a digital table (see Fig. 7) and a large high-resolution wall display. The table allowed people to explore the visualization via direct-touch, while interaction with the wall display was supported through a mouse. At the public library, people explored the same early W@nderverse prototype on laptops. The events were advertised across the campus, at the public library and in town. While we were present at both events and provided some background about the project, people interacted with the visualization largely unsupervised in a walk-up-and-use fashion.

Participants. Approx. 50+ people of varying backgrounds and interests attended the events. 27 participants provided feedback about their experiences with the Speculative W@nderverse through the questionnaires. Their background was diverse, with five people working in computer science (three with backgrounds in HCI and InfoVis), eleven researchers in English Literature and Creative Writing, two people with a Library Science background, two focusing on Communications, and one Archaeologist; six people did not specify their background. In general, we can classify this audience as highly educated, consisting mostly of students, academics, or knowledge workers. $62 \%$ of our participants were between 26 and 45 years old; $22 \%$ (6 people) were older and $14 \%$ (4 people) younger than this.

Data Collection \& Analysis. In our questionnaires we asked about people's background, what types of discoveries they made through the W@nderverse (if any), and which aspects of the visualization they liked/disliked and why. We also asked for suggestions on additional features, and if they could imagine using the tool in the future. We analyzed participants' answers through an open-coding approach, wherein categories of codes evolved through the questions themselves.

\subsection{In-Team Longterm Evaluation}

An essential part of evaluating the Speculative W@nderverse was to study how literary scholars would use it as part of their research and what kind of insights and discoveries it can support (if any). This type of evaluation requires the long-term study of domain experts: literary research, much like any other form of research, requires time before insightful results can be formulated. While research on science fiction literature is an active field, to date, the only scholars who focus on the Gibson Anthologies are the two scholars in our research team. Consequently, they are the only suitable domain experts to help evaluate the W@nderverse's potential for supporting in-depth literary research processes. We therefore came up with a methodology to keep track of our colleagues' research process and progress throughout the project. This approach comes close to what Neustaedter and Sengers have called "autobiographical design", that is, "design research drawing on extensive, genuine usage by those creating or building a system" [30] While this approach has obvious limitations, we highlight that the goal of our empirical study is to present examples of public and academic practices around visualizations of literary collections that are likely to transfer into other humanities contexts.

Data Collection \& Analysis. Over a period of 1.5 years, the two literary scholars tracked any thoughts, assumptions, research questions, and discoveries that came up during their archival work with the anthologies, their extraction of metadata, and their exploration of the visualization prototypes in the form of written notes that were shared with the whole team. The resulting document also reflected thoughts that came up during our in-team discussions, which took place once a month in the first year of the project and every two weeks as the visualization design stabilized and went through more rapid, fine-grained iterations. As part of these meetings we would discuss the progress of the visualization, new discoveries and research questions that had come up, and potential visualization features to address these.

It is important to note that the design and development of the visualization was paralleled by the manual extraction of metadata from the anthologies, that is, the visualization evolved not only in terms of new features but also in terms of its underlying data. While the data extraction progressed, our scholars slowly intensified their work with the W@nderverse, using it to investigate their initial research questions and looking for ways of interpreting answers that the visualization would offer. Through this process, general themes crystallized that show how the visualization integrated into and, indeed, changed the scholars' research processes and their perspective on the collection,

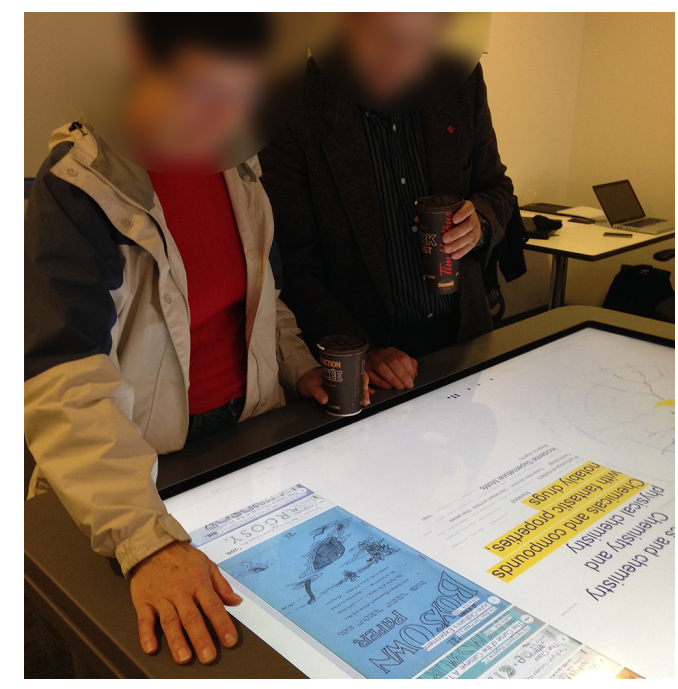

Fig. 7: Visitors exploring the Speculative W@nderverse at a multitouch table at our open house. 
while limitations of the visualization also became apparent.

This two-part empirical study of the Speculative W@nderverse with a public audience and literary scholars provides detailed insights into the role that visualization can play in facilitating the exploration of complex, untapped literary collections.

\section{EXPloration Processes \& Discoveries}

In the following paragraphs we describe how our general-interest audience and our literary experts explored the Speculative W@ nderverse and the types of discoveries they made.

\subsection{Public Response}

People's reactions to the Speculative W@ nderverse were very positive. Of the 27 people who provided feedback, $74 \%$ felt they had learned something from the visualization, $96 \%$ thought they understood the represented information, $92 \%$ thought the visualization was easy to interact with, and $96 \%$ would use it in a personal and/or professional context. Participants' statements show that these positive responses have to do with the discoveries and lightweight contextual access to the Gibson Anthologies enabled by the W@nderverse.

\subsubsection{Discoveries \& Insights}

Interaction times with the visualization were diverse, ranging from 2 20 minutes. Some people came back several times for repeated interactions. No matter how long people interacted, most were able to draw some insights from the W@ nderverse: all but one participant described discoveries they had made about the anthologies, which can be categorized into high- and low-level insights.

High-level insights describe general insights that people gathered from the visualization. For example, participants stated that they learned about the "range of topics in the anthologies." [p8, Librarian] and "the overlapping areas collected; the vast richness of the Gibson collection" [p10, Medieval Literature]. Most people were simply amazed by the diversity of the collection; "the extent to which one person can create such an extensive collection." [p21, Communication].

People were also able to gather more low-level details about specific aspects of the anthologies through the W@ nderverse. One person stated: "The keywords themselves are very intriguing! For example, 'glassed over cities', 'living brains in jars'-makes me curious about these stories." [p12, InfoVis]. Another participant found out about "The influence of astronomy on early SciFi and that voodoo was relevant in this literature" [p23, English Lit.]. People also discussed particular stories they found through their explorations: "There was a story about 'living hair'." [p4, Comp. Sci.]. These statements illustrate that even after brief interaction times, the visualization conveyed general insights about our sub-sample of anthologies, as well as finegrained details about specific themes and Gibson items. The Keyword Cloud and Item List played an important role in these discoveries, as both visualization elements make topics and items included in the anthologies readily accessible in a familiar way. The Hierarchy View was also frequently explored but required more explanation.

\subsubsection{Enabling Access \& Sparking Interest}

People's comments indicate that the W@nderverse not only supported easy access to the anthologies, but that it also promoted curiosity and genuine interest in the collection and its content, both of which were previously unknown to most visitors at our openings: "I was delighted to discover a dauntingly vast collection rendered 'accessible' [through the visualization]." [p5, English Lit.], "The collection immediately takes on a new connotation with this visualization. It's immediately more accessible and consequently more attractive." [p18, English Lit.], and "Really engaging combo of content and vis! I could sit here for a long time!" [p12, InfoVis].

Metadata as Entry Points. The visual overviews provided by the Keyword Cloud, Hierarchy View, and Symbol Cluster, as well as the low-level content and visual imagery which characterizes the Item List, act as entry points to this complex collection. People also indicated that the interactive visual filtering options based around topics and time periods were useful: "I liked how quickly you could narrow down a range of story content by keywords. [p22, English Lit.].
Aesthetic Aspects of the Collection. Not surprisingly, the unique covers of the anthologies also sparked interest, and people appreciated their prominent role in the visualization: "My favourite part is the cover snippets - they are beautiful \& have character." [p12, InfoVis] and "I particularly liked the inclusion of Gibson's cover drawings in the right-hand margin." [p17, English/Creative Writing]. However, a lot of people suggested that we further emphasize material and visual qualities of the anthologies: "Some alternate representation of the anthologies as artifacts. I think it is important that this is still visualized, as Gibson created these [the anthology covers] as a way to understand the content." [p1]. This echoes the importance of reflecting on a collection's material and visual qualities through its visualization.

Participants' comments clearly indicate that the visualization raised interest for actual stories, particular authors, and specific topics included in the Gibson Anthologies. The visualization prototype we presented at the open house events did not support direct access to the digitized stories, but people were yearning for this: "I really want to read the material/content now!" [p7] and "Looking forward to access to the anthologies." [p27]. Five participants explicitly suggested the inclusion of direct links to the full stories in the visualization.

The Gibson Anthologies are uniquely poised to raise people's interest, in part because of their quirky material and visual aesthetic, which arises from their handcrafted construction by a single collector, and because of their science fiction content, which is of interest to scholars and general-interest readers. Even if this collection was readily accessible in physical or digital form, people could not easily get an overview of what it is about and what may be of interest to them. As the comments from our open house events show, a visualization such as the Speculative W@nderverse can provide this type of lightweight accessibility that promotes more in-depth content exploration.

\subsubsection{Possible Usage Scenarios}

All except one participant stated that they could imagine using the Speculative W@nderverse in other contexts, ranging from personal to professional scenarios. For personal enjoyment, several people wanted to focus on the anthology covers: "Mainly to enjoy the cover art!' [p11, HCI]. Others appreciated the visualization for finding stories: "I like reading fiction, and I would like to be able to read vintage works in specific topics of my interest." [p26] and "I could use it for searching for reading material (for personal use), for example, fairy tales." [p26, Archaeology]. Other people stated that they would like to browse the visualization for "inspiration" [p25, English Lit].

15 participants could see themselves using the W@nderverse in a professional context. For example, some English Literature scholars were generally interested in women writers, others in particular authors: "[...] particularly, I'd be interested in quantifying female authors who were writing subversive lit during the period" [p9, English Lit.]. "I study Lovecraft and his circle. Would love to pour through this." [p16, English Lit.]. People could also see the relevance of the anthologies to other topics of professional interest: "Relating [Gibson items] to different contexts of academic trends, possibly in urban systems.” [p23, English Lit + Urban Studies]. Three participants mentioned that they would like to use the W@ nderverse for teaching and to promote the Gibson Anthologies beyond the university. These statements indicate the relevance of the Gibson Anthologies to other (research) areas beyond science fiction literature, but they also show that the visualization highlights the diverse content of the collection and communicates the anthologies' relevance to a wide audience.

Some scholars openly admitted that they were skeptical of the use of computational tools to facilitate literary research. The Speculative W@nderverse converted at least one of them: “It's amazing! I could see myself using it. Can see utility of digital humanities through this.” [p16, English Lit.]. Another scholar expressed the idea of using the W@nderverse as "an example for planning other digitization projects.” [p15, Library \& Information Studies].

The positive feedback and the value people can see in the visualization for different scenarios, even after brief explorations of the anthologies through the W@ nderverse, are highly encouraging. However, we were also interested in how the visualization could support research-driven explorations of the Gibson Anthologies. 


\subsection{Explorations and Discoveries by Literary Scholars}

The literary scholars in our team used the Speculative W@nderverse in combination with their traditional research approaches. This led to substantial discoveries regarding the meaning of Gibson symbols, the role of female authors in early science fiction, and genre evolution. A detailed description of these findings is beyond the scope of this paper. Instead, we summarize the ways in which the W@ nderverse supported literary research processes based on selected examples.

Confirming Hypotheses. Informed by their research background and their archival work with the collection, our scholars had several hypotheses about the evolution of the science fiction genre and the meaning of some of the Gibson symbols. Interaction with the different views of the W@nderverse helped them to confirm and substantiate these hypotheses. For example, the visualization confirmed a widely held view in science fiction research which claims that modern science fiction begins to takes shape in the 1880s and 90s-the visualization shows a clear peak of Gibson items during this time. The W@nderverse has also been useful for verifying our scholars' hypothesis about the meanings of several Gibson symbols, including the "dot" symbols, which signify a ranking system, wherein more dots mark higher-quality science fiction content (as evaluated by Gibson).

Refining Research Questions. The visualization helped our scholars to refine their initially broad and open-ended research questions. The overviews of different aspects of the anthologies, in combination with the filtering mechanism, promoted narrowing and (re-)defining these questions in a way that was directly guided by the collection's characteristics. For example, our scholars were initially interested in what a fan's collection could reveal about the history of science fiction. The different views helped to break down this open-ended question. For example, the scholars investigated the presence of certain motifs in the collection using the Hierarchy View and Keyword Cloud, they zoomed in on items written in the late 19th century using the timeline, and they explored the frequency and names of female authors in this same time period using the gender filter mechanism. Interacting with the visualization showed them that any answers to their questions work within the parameters (and limitations) of the collection itself, while helping to identify these parameters.

New Ways of Answering Research Questions. Our scholars were pleasantly surprised to find that the W@nderverse also suggested new ways of answering some of their research questions. The visualization highlights relationships between symbols, items, authors, and source periodicals. This explicit and visual intersection of metadata triggered our scholars to ask about the role of specific periodicals in shaping different kinds of 19th-century science fiction and its motifs. Before using the visualization, our scholars had not thought of approaching their main research question through this lens. The different visual overviews shown side-by-side triggered the idea and prompted further explorations. We highlight here how a visualization such as the Speculative W@ nderverse can promote such thinking processes.

\section{Negotiating Quantitative \& Qualitative Interpretation.} Visualization-based research in the humanities has to integrate effectively with archival work and close reading. We found that the W@nderverse enabled fluid transitions from high-level qualitative questions nourished by archival work and the study of Gibson stories, to a more quantitative analysis. For example, the assumption that the "dot" symbols correspond to a rating of science fiction content was substantiated by the fact that there are far more items with one or two dots than there are items with three or four-high-quality items are more rare. The Symbol Cluster immediately highlights these differences. In turn, quantitative information can directly trigger a need for qualitative interpretations. For example, when exploring the meaning of certain symbols (e.g., the stylized "JF" symbol, which, as our scholars discovered, likely stands for "Juvenile Fiction", see Fig. 6), the visualization helped to identify not only the number of associated items but also the motifs to which they correspond (the supernatural, magic, and mystical animals). In turn, the archival work and study of Gibson items helped to further develop hypotheses and plausible interpretations that triggered revisions of the tool and new visualization ideas. For example, our scholars returned to the archive to document the material characteristics of the anthologies to explore what the anthologies can reveal about Gibson's process of making. This led to a brainstorming ways in which these material features could be incorporated into the visualization and explored in the future.

New Research Questions \& Areas. Work with the W@nderverse in combination with archival work, led to the identification of new research questions and areas for future work, showing how visualizations can foster a dynamic research process. For example, what is unique about the formative years of science fiction (triggered by the discovery of certain source magazines in the 1880s and 90s, authors, and associated keywords)? Which aspects of early science fiction survive, which are eliminated, and when do these changes take place (triggered by the different motifs visible in the Hierarchy View in relation to the timeline)? The visualization also highlights the limitations of the Bleiler keywords, which do not always adequately apply to Gibson items. In the future, we will explore the use of free-form (possibly crowdsourced) keywords to further characterize the anthologies.

\section{Discussion}

Our findings show that a visualization such as the W@nderverse can promote curiosity and several entry points into a large and unknown literary collection among general-interest readers, while fostering a dynamic research process, inviting literary scholars to explore initial assumptions and research questions. The Speculative W@nderverse therefore illustrates one interpretation of the design space of visualization as part of literary research that we propose in this paper. In the following, we critically discuss our approach in the context of this design space and the questions it raises.

From Early Explorations to In-Depth Vis Experiments. The visualization has proven valuable to both general-interest readers and literary scholars. The former were drawn particularly toward the thematic overviews provided by the Keyword Cloud and the anthology covers and individual items that are visible through the Item View. In contrast, the coordinated views and filters that enable dynamic (visual and quantitative) experiments with the collection helped our scholars to explore their initial research questions, to verify assumptions, and to identify new and intriguing research questions and topics suggested by the anthologies through their metadata, as well as their material and visual qualities. Regarding the audience dimension of our proposed design space, the W@nderverse renders a complex literary collection visible and makes it accessible and explorable from different perspectives, promoting engagement from multiple audiences. This approach (1) bridges the gap between academics, fans, and general-interest audiences who can learn from and inform each other's insights, (2) it can help to identify shared interests among academics and expert amateurs for interdisciplinary collaborations, and (3) it may demonstrate the benefits of digital tools to skeptical humanities' scholars.

Considering the design and usage needs and expectations of both general-interest audiences and scholars is a valuable approach in early stages of literary visualization projects, to elicit feedback and explore visualization ideas that form around the collection in focus. This is particularly true for projects that focus on largely unexplored collections containing unknown material or collections of known literary works, which develop new meanings and connotations through adjacencies with other works. While this approach nicely supports and is invaluable to inform initial (scholarly) explorations, it cannot replace more specialized (potentially separate) visualization experiments that cater to complex, in-depth questions and analyses that scholars will inevitably develop through their interactions with visualizations such as the W@nderverse. The W@nderverse can therefore be thought of as a versatile, yet broad, lens to the Gibson Anthologies, that enables initial explorations. However, through these very explorations, the limitations and boundaries of this lens become apparent, which, in turn, can inform the design of further visualization experiments.

Combining Quantitative Analysis \& Literary Interpretation. Our case study illustrates how visualization approaches in the context of literary studies can productively address seemingly opposite methods of quantitative analysis vs. interpretation. The W@ nderverse visualization directly shows quantitative aspects of the anthologies, e.g., 
the temporal distribution, female vs. male authors, and frequency of particular symbols. However, our very choice of what metadata to represent through the visualization and the filters we included are a direct reflection of our initial interest in the Gibson Anthologies.

This qualitative interpretation of the Anthologies further manifests itself visually, e.g., through our choice of representing Bleiler's keyword hierarchy in a circular rather than in a vertical layout. As described earlier, this choice directly reflects on our intention to let the anthologies' content derive visual emphases instead of implying a hierarchy or structure within the motifs through layout. While some previous work has utilized visual representations as an act of interpreting the underlying data collection (e.g., [21]), many visualization case studies in the humanities mainly focus on the quantitative aspects of the collection in focus. We encourage researchers in visualization and digital humanities to explore actively how to utilize visualization as a means to reflect on literary interpretations while featuring methods of quantitative analysis. Depending on the collection, existing visualization techniques may already support such interpretations (just like the Hierarchy View in our case), but interpretative approaches may also inspire the invention of new techniques specific to the collection. For example, the use of visual metaphors can reflect the character of the cultural collection (see [21]). We will explore such approaches in the future, including the question of how to visually reflect underlying interpretations through visualization.

Enabling Fluid Interrogation and Experimentation. Our case study shows that visualization can support a wide range of research approaches to exploring a cultural collection such as the Gibson Anthologies. Our use of multiple coordinated visual perspectives enables fluid movements between high-level trends and specific details. These views can also be considered as a reflection on the speculative nature of the collection and our interpretative approach, which tempers the visualization's quantitative features (quantitative analysis vs. interpretation). Although the explorations of our scholars were driven by research questions, the visual views invited curiosity-driven experiments, where our scholars manipulated certain filters (e.g., gender or time) just to see how the views would change. This fluid interrogation of the collection led to unexpected discoveries about the anthologies and inspired the addition of new search and filter functionalities. The ways in which the visualization changed our scholars' perspective on the Gibson Anthologies can be considered a big success [41].

Building upon previous work $[11,45,46,53]$, our case study emphasizes the positive influence of multiple coordinated views in the context of visualizing literary collections: offering these perspectives facilitates the exploration of the collection from multiple aspects and promotes an open and curious mindset to trigger questions beyond what is being presented.

Promoting Visualization as a Process. Recent scholarship has begun to discuss visualization as a starting point to humanities research, rather than a means to an end [20]. We approached this idea by introducing visualization prototypes early on in the literary research process, while our scholars were still heavily engaged in archival work and the manual extraction of metadata. In this way insights gradually developed both through archival work and the experimentation with the evolving visualization. This intertwined work process stimulated thinking and re-thinking the collection's characterizing features and how to visualize these effectively. The scholars' exploration of one visualization prototype would instigate questions that would make new iterations necessary- limitations not only of particular functionalities would become apparent but also conceptual flaws, e.g., the lack of anthologies' material and visual aspects. Encountering these limitations, in turn, inspired discussions on new design iterations, some of which have to be addressed through entirely new visualizations.

Along these lines, we advocate for visualization as a process that can be incorporated into literary research at multiple points (visualization as a result vs. process). This questions approaches where decisions about data abstraction and visual representation are separated from visual explorations themselves. However, more work is necessary to explore how to support this fluid process of developing and modifying visualizations while new insights and perspectives on the underlying literary collection are evolving. Easily adaptable visualization tools, while useful to a certain extent, introduce their own visual and conceptual constraints, which may negatively influence and even hamper qualitative literary interpretation. Collaborations between visualization experts and literature scholars that enable iterative work on customized visualization are resource intensive and require time. We therefore propose to investigate how to facilitate interdisciplinary collaborative processes between humanities scholars and visualization experts. In particular we need to explore lightweight yet versatile mechanisms that provide a platform for visualization experts and humanities researchers to experiment with and critically discuss visualization ideas and approaches throughout the collaborative process. Here, constructive visualization comes to mind, a design method introduced by Huron et al. to enable non-experts (e.g., people who have little experience in developing visualizations) to create and modify representations of their data [22]. Future work could explore how to apply this method as part of projects at the intersection of InfoVis and literary studies (if not the humanities at large).

Sensitivity to Material \& Visual Qualities. Visitor reactions at the open house events indicate that it was to a large extent the visibility of the anthology cover images in the Item View that triggered curiosity in and engagement with this collection. As Galey states: "The capacity to inspire should not be underestimated when we evaluate digital objects" [16]. However, as our scholars highlighted, the importance of reflecting on the material through the visualization, i.e., both physical and visual qualities of the anthologies, goes beyond this engagement aspect. In particular with this handcrafted collection, the material and visual qualities provide a context that is crucial for interpretation: it is not only the content but also its presentation that informs and, in fact, drives analysis and interpretation. Many literary and cultural collections have similar unique characteristics manifested in their material and visual forms. With the Speculative W@nderverse we are only scratching the surface of what can be done to integrate these qualities into a visualization. Previous projects have started to explore this space $[21,46]$, but there is more to be done. For example, image processing methods could be applied to generate material-based metadata of cultural collections, which could then be represented through visual variables, such as original textures, fonts or imagery, to overcome and prevent otherwise reduced and impoverished impressions of the visualized collection. We therefore end with a call for a more creative take on the visualization of cultural collections that considers their unique material and visual identities. Cultural collections contain pieces of art and, as such, corresponding visualization tools should be no less: an artistic interpretation of and response to the collection.

\section{Conclusion}

We have presented the Speculative W@nderverse as a case study that shows how visualization can integrate into literary studies' research processes while supporting casual explorations by general-interest readers. As part of this, we have outlined the design space of InfoVis in this context which is defined by an audience that ranges from generalinterest readers, to fans as amateur experts, to academics, an approach that reflects a tension between quantitative analysis and qualitative interpretation of literary collections, the role of visualization as part of humanities research (result vs. process), and the material and visual qualities of a literary collection as a pivotal aspect to interpretation. Based on two empirical studies, we have presented the benefits and types of insights that visualizations such as the W@nderverse can provide for general-interest readers and academics, and critically discussed our approach to facilitating literary research processes through visualization and the future research questions this raises.

\section{ACKNOWLEDGMENTS}

We would like to thank all graduate students who helped annotate the Gibson Anthologies. We also thank the Faculty of Arts at the University of Calgary for supporting our open house events and all participants for providing invaluable feedback to our research. Furthermore, we thank Jason Reid and John Brosz for their technical support with the Gibson database, and, last but not least, SSHRC for funding this research. 


\section{REFERENCES}

[1] M. Bingenheimer, J. Hung, and S. Wiles. Social Network Visualization from TEI Data. Literary and Linguistic Computing, 26(3):271-278, 2011.

[2] E. Bleiler. Science Fiction: The Early Years. Kent State University Press, 1990.

[3] F. Block, M. S. Horn, B. C. Phillips, J. Diamond, E. M. Evans, and C. Shen. The DeepTree Exhibit: Visualizing the Tree of Life to Facilitate Informal Learning. IEEE TVCG, 18(12):2789-2798, 2012.

[4] M. Boyd. Library Collections and Archives of SF and Related Materials. Science Fiction Studies, 37(2):180-181, 2010.

[5] M. Brehmer, S. Ingram, J. Stray, and T. Munzner. Overview: The Design, Adoption, and Analysis of a Visual Document Mining Tool For Investigative Journalists. IEEE TVCG, 20(12):2271-2280, 2014.

[6] S. Brown, S. Ruecker, J. Antoniuk, S. Farnel, M. Gooding, S. Sinclair, M. Patey, and S. Gabriele. Reading Orlando with the Mandala Browser: A Case Study in Algorithmic Criticism via Experimental Visualization. Digital Studies, 2(1), 2010.

[7] T. Clement. Distant Listening or Playing Visualisations Pleasantly with the Eyes and Ears. Digital Studies. Digital Studies, 3(2), 2012

[8] T. E. Clement. 'A thing not beginning and not ending': using digital tools to distant-read Gertrude Stein's The Making of Americans. Literary and Linguistic Computing, 23(3):361-381, 2008.

[9] C. Collins, F. Viégas, and M. Wattenberg. Parallel Tag Clouds to Explore Faceted Text Corpora. In Proc. of IEEE VAST, pages 91-98, 2009.

[10] A. Don, E. Zheleva, M. Greogry, S. Tarkan, L. Auvil, T. Clement, B. Shneiderman, and C. Plaisant. Discovering Interesting Usage Patterns in Text Collections: Integrating Text Mining with Visualization. In Proc. of Information Knowledge and Management, pages 213-222, 2007.

[11] M. Dörk, S. Carpendale, C. Collins, and C. Williamson. VisGets: Coordinated Visualizations for Web-based Information Exploration and Discovery. IEEE TVCG, 14(6):1205-1212, 2008.

[12] M. Dörk, S. Carpendale, and C. Williamson. The Information Flaneur: A Fresh Look at Information Seeking. In Proc. of CHI, pages 1215-1224, 2011.

[13] J. Drucker. Humanities Approaches to Graphical Display. Digital Humanities Quarterly (DHQ), 5(1), 2011.

[14] E. K. et al. Digging into Data White Paper: Trading Consequences. Technical report, University of Edinburgh, University of St Andrews, University of York, University of Saskatchewan, 2014.

[15] P. S. et al. Thinking Around the Box: the Work of the Bethan Project. British Academy Review, 13, 2009. https://www.ucl.ac.uk/BenthamProject/documents/brit_acad_rev.

[16] A. Galey and S. Ruecker. How a Prototype Argues. Literary and Linguistic Computing, 25(4):405-424, 2010.

[17] F. Gibbs and T. Owens. Building Better Digital Humanities Tools: Toward Broader Audiences and User-Centered Designs. Digital Humanities Quarterly (DHQ), 6(2), 2012.

[18] C. Görg, Z. Liu, J. Kim, J. Choo, H. Park, and J. Stasko. Combining Computational Analysis and Interactive Visualization for Document Exploration and Sensemaking in Jigsaw. IEEE TVCG, 19:1646-1663, 2014.

[19] N. K. Hayles. How We Think: Digital Media and Contemporary Technogenesis, chapter The Digital Humanites, pages 23-54. The University of Chicago Press, 2012.

[20] U. Hinrichs, B. Alex, J. Clifford, and A. Quigley. Trading Consequences: A Case Study of Combining Text Mining \& Visualisation to Facilitate Document Exploration. In Proc. of Digital Humanities, 2014.

[21] U. Hinrichs, H. Schmidt, and S. Carpendale. EMDialog: Bringing Information Visualization into the Museum. IEEE TVCG, 14(6):1181-1188, 2008.

[22] S. Huron, S. Carpendale, A. Thudt, A. Tang, and M. Mauerer. Constructive Visualization. In Proc. of DIS, pages 433-442, 2014.

[23] S. Jänicke, G. Franzini, M. Cheema, and G. Scheuermann. On Close and Distant Reading in Digital Humanities: A Survey and Future Challenges. In Proc. of EuroVis-STARs, pages 83-103, 2015.

[24] H. Jenkin. Confronting the Challenges of Participatory Culture. The MIT Press, 1958

[25] M. Jockers. Macroanalysis: Digital Methods and Literary History. University of Illinois Press, 2013

[26] S. Marche. Literature is not Data. Los Angeles Review of Books http://lareviewofbooks.org/essay/literature-is-not-data-againstdigital-humanities, 2012. accessed July 2015.

[27] F. Moretti. Graphs, Maps, Trees: Abstract Models for a Literary History.
Verso, 2007.

[28] F. Moretti. Distant Reading. Verso, 2013.

[29] T. Nagel, M. Maitan, E. Duval, A. V. Moere, J. J. Klerkx, K. Kloeckl, and C. Ratti. Touching Transport - A Case Study on Visualizing Metropolitan Public Transit on Interactive Tabletops. In Proc. of AVI, pages 281-288, 2014.

[30] C. Neustaedter and P. Sengers. Autobiographical Design in HCI Research: Designing and Learning through Use-It-Yourself. In Proc. of DIS, pages 514-523, 2012.

[31] C. Plaisant, B. Yu, M. G. Kirshenbaum, J. Rose, L. Auvil, M. N. Smith, T. Clement, and G. Lord. Exploring erotics in Emily Dickinson's correspondence with text mining and visual interfaces. In Proc. of JCDL, pages 141-150, 2006.

[32] S. Posavec. Writing Without Words. http://www.stefanieposavec.co.uk/writing-without-words. Accessed July 2015.

[33] Z. Pousman, J. T. Stasko, and M. Mateas. Casual Information Visualization: Depictions of Data in Everyday Life. IEEE TVCG, 13(6):11451152, 2007

[34] T. S. Presner. Hypermedia Berlin: Cultural History in the Age of New Media or "Is There a Text in this Class?". Vectors 1.2 http://vectorsjournal.org/projects/index.php?project=60, 2006. Accessed July 2015.

[35] S. Ramsay. Algorithmic Criticism, chapter Algorithmic Criticism. A Companion to Digital Literary Studies. Ed. Susan Schreibman and Ray Siemens, pages 479-91. Oxford: Blackwell, 2008.

[36] E. M. Reingold and J. S. Tilford. Tidier Drawings of Trees. IEEE Transactions on Software Engineering, 7(2):223-228, 1980.

[37] G. Rockwell, S. Sinclair, K. Uszkalo, and M. Radzikowska. TAPoR. http://www.tapor.ca/, 2014. accessed March 2015.

[38] S. Ruecker, M. Radzikowska, P. Michura, C. Fiorentino, and T. Clement. Visualizing Repitition in Text. Digital Studies, 1(3), 2009.

[39] J. Schnapp and T. Presner. The Digital Humanities Manifesto 2.0. http://manifesto.humanities.ucla.edu/2009/05/29/the-digitalhumanities-manifesto-20/, 2006. accessed March 2015.

[40] J. Schreibman and A. Hanlon. Determining Value for Digital Humanities Tools: Report on a Survey of Tool Developers. Digital Humanities Quarterly $(D H Q), 4(2), 2010$.

[41] M. Sedlmair, M. Meyer, and T. Munzner. Design Methodology: Reflections from the Trenches and the Stacks. IEEE TVCG, 18(12):2431-2440, 2012

[42] S. Sinclair. Meetings with Books: Special Collections in the 21st Century, chapter Thinking Bigger: Reflections on the Digital, pages 125-132. McGill University Library and Archives, 2014.

[43] S. Sinclair and G. Rockwell. Voyant Tools. http://voyant-tools.org/. accessed July 2015

[44] S. Sinclair, S. Ruecker, and M. Radzikowska. Information Visualization for Humanities Scholars. MLA Commons https://dlsanthology.commons.mla.org/information-visualization-forhumanities-scholars/. accessed July 2015.

[45] J. Stasko, C. Görg, Z. Liu, and K. Singhal. Jigsaw: Supporting Investigative Analysis through Interactive Visualization. In Proc. of VAST, pages $118-132,2007$.

[46] A. Thudt, U. Hinrichs, and S. Carpendale. The Bohemian Bookshelf: Supporting Serendipitous Discoveries through Information Visualizaiton. In Proc. of CHI, 2012.

[47] L. Udsen and A. Jøgensen. The Aesthetic Turn: Unravelling Recent Aesthetic Approaches to Human-Computer Interaction. Digital Creativity, 16(4):205-216, 2005.

[48] F. van Ham, M. Wattenberg, and F. B. Viégas. Mapping Text with Phrase Nets. IEEE TVCG, 15(6):1169-1176, 2009.

[49] F. Viégas, M. Wattenberg, F. van Ham, J. Kriss, and M. McKeon. Many Eyes: A Site for Visualization at Internet Scale. IEEE TVCG, 13(6):1077-2626, 2007.

[50] F. B. Viégas and M. Wattenberg. Tag Clouds and the Case for Vernacular Visualization. Interactions, 15(4):49-52, 2008

[51] F. B. Viégas, M. Wattenberg, and J. Feinberg. Participatory Visualization with Wordle. IEEE TVCG, 15(6):1137-1144, 2009.

[52] M. Wattenberg and F. B. Viégas. The Word Tree, an Interactive Visual Concordance. IEEE TVCG, 14(6):1221-1228, 2008

[53] C. Weaver. Multidimensional Visual Analysis Using Cross-Filtered Views. IEEE TVCG, 16(2):192-204, 2009.

[54] M. Whitelaw. Generous Interfaces for Digital Cultural Collections. Digital Humanities Quarterly (DHQ), 9(1), 2015. 